\title{
Exchange energy in the local Airy gas approximation
}

\author{
Vitos, Levente; Johansson, B.; Kollár, J.; Skriver, Hans Lomholt
}

Published in:

Physical Review B Condensed Matter

Link to article, DOI:

10.1103/PhysRevB.62.10046

Publication date:

2000

Document Version

Publisher's PDF, also known as Version of record

Link back to DTU Orbit

Citation (APA):

Vitos, L., Johansson, B., Kollár, J., \& Skriver, H. L. (2000). Exchange energy in the local Airy gas approximation. Physical Review B Condensed Matter, 62(15), 10046-10050. https://doi.org/10.1103/PhysRevB.62.10046

\section{General rights}

Copyright and moral rights for the publications made accessible in the public portal are retained by the authors and/or other copyright owners and it is a condition of accessing publications that users recognise and abide by the legal requirements associated with these rights.

- Users may download and print one copy of any publication from the public portal for the purpose of private study or research.

- You may not further distribute the material or use it for any profit-making activity or commercial gain

- You may freely distribute the URL identifying the publication in the public portal

If you believe that this document breaches copyright please contact us providing details, and we will remove access to the work immediately and investigate your claim. 


\title{
Exchange energy in the local Airy gas approximation
}

\author{
L. Vitos and B. Johansson \\ Condensed Matter Theory Group, Physics Department, Uppsala University, S-75121 Uppsala, Sweden \\ J. Kollár \\ Research Institute for Solid State Physics, H-1525 Budapest, P.O. Box 49, Hungary \\ H. L. Skriver \\ Center for Atomic-scale Materials Physics and Department of Physics, Technical University of Denmark, DK-2800 Lyngby, Denmark
}

(Received 14 April 2000)

\begin{abstract}
The Airy gas model of the edge electron gas is used to construct an exchange-energy functional that is an alternative to those obtained in the local-density and generalized-gradient approximations. Test calculations for rare-gas atoms, molecules, solids, and surfaces show that the Airy gas functional performs better than the local-density approximation in all cases and better than the generalized-gradient approximation for solids and surfaces.
\end{abstract}

Since the pioneering papers on density-functional theory $^{1,2}$ (DFT) there has been a constant search for exchange-correlation functionals of chemical accuracy. This includes the works on the generalized-gradient approximation $^{3-7}$ (GGA) that are dedicated efforts to construct local functionals for inhomogeneous systems ranging from atoms to solids based on the uniform electron gas, i.e., the local-density approximation (LDA), and density-gradient corrections, as well as the development of a number gradient level and semiempirical functionals. ${ }^{8-11}$ The GGA functionals have had considerable impact upon the fields of quantum chemistry and solid state physics because they reduce the LDA overbinding and generally improve the calculated properties, relative to experiments, of molecules ${ }^{5,12-14}$ and bulk solids. ${ }^{15-20}$ However, they perform less well for the bulk properties of late transition metals and semiconductors, ${ }^{21-23}$ and the underestimate of the exchange energies of surfaces ${ }^{15}$ as well as the overestimate of the dissociation energies of the multiply bonded molecules ${ }^{5,13}$ indicates the necessity to go beyond the gradient level approximations and develop functionals that depend upon other inhomogeneity parameters, e.g., higher derivatives of the charge density or the KohnSham kinetic energy density. One step in this direction is the meta-generalized-gradient approximation (meta-GGA) of Perdew, Kurth, Zupan, and Blaha, ${ }^{12}$ which proves highly promising for both finite and extended systems. ${ }^{13}$

In the present work we introduce and apply a gradientlevel exchange-energy functional based on the concept of the edge electron gas. ${ }^{24}$ Besides the formal interest in the development of density-based, orbital independent functionals, there are several reasons why in applications of DFT the focus is on the approximate, local exchange-correlation schemes. Within the Kohn-Sham approach to DFT the KohnSham exchange energy may be determined exactly, and as demonstrated recently ${ }^{25-27}$ so may the corresponding local exchange potential. However, the exact Kohn-Sham exchange formalism is nonlocal and orbital-based, i.e., both the exchange energy and potential are highly complicated nonlocal functionals of the Kohn-Sham orbitals. In consequence, the application of exact exchange is computationally demanding. Furthermore, when exchange is treated exactly the error cancellations between the exchange and correlation energies on which all approximate schemes depend are lost owing to the poor description of correlation effects and, as a result, the total energies worsen. ${ }^{15,26}$ For these reasons the exact Kohn-Sham exchange energy has only been used in practice in connection with semiempirical, hybrid approximations. $^{28}$

The concept of the edge electron gas was put forward by Kohn and Mattsson ${ }^{24}$ as an appropriate basis for the treatment of systems with edge surfaces outside of which all Kohn-Sham orbitals decay exponentially. Its simplest realization, the Airy gas model, is based on the linear potential approximation and may serve as the starting point for the construction of functionals that are alternatives to the GGA. The Airy gas model has recently been used to construct an explicit kinetic energy functional for inhomogeneous systems, ${ }^{29}$ which for atoms and surfaces has the accuracy of functionals based on a second-order gradient expansion.

Here we have taken the exchange energy of the Airy gas model derived by Kohn and Mattsson ${ }^{24}$ and cast it in a form amenable to a simple, accurate parametrization. The procedure may be viewed as local mapping of the real system described by its density and scaled gradient onto the Airy gas model and represents one possible solution to the joining of the interior to the edge regions. The parametrized functional that we refer to as the local Airy gas (LAG) functional is tested in calculations of the exchange energies of rare-gas atoms and of metallic surfaces within the jellium model where the exact results are known. ${ }^{30}$ In addition, we apply the LAG exchange functional in conjunction with the LDA for correlation ${ }^{31}$ in calculations of the molecular binding energies and bulk properties of solids.

The present LAG exchange functional has a number of advantages over previous GGA functionals: (i) it explicitly includes the properties of the edge region where much interesting physics occurs, (ii) its accuracy may be systematically improved by including higher-order expansions of the effec- 
tive potential of the model system, and (iii) the resulting exchange-energy functional is as simple and well-defined as that of the standard LDA, i.e., it has no adjustable parameters.

The starting point for the Airy gas exchange energy functional is the potential

$$
v_{e f f}(z)= \begin{cases}\infty & \text { for } \quad z \leqslant-L \\ F z & \text { for } \quad-L<z<\infty,\end{cases}
$$

which is linear in $z$, independent of $x$ and $y$, and has a hard wall at $-L$ far from the electronic edge at $z=0$. The slope of the effective potential $F=d v_{e f f} / d z$ leads to a characteristic length scale

$$
l \equiv\left(\frac{\hbar^{2}}{2 m F}\right)^{1 / 3},
$$

and the electron and exchange-energy densities are then given by

$$
n(z)=l^{-3} n(\zeta)
$$

and

$$
\varepsilon_{x}(z)=-\frac{e^{2}}{2} l^{-4} \varepsilon_{x}(\zeta)
$$

where $\zeta=z / l$,

$$
n(\zeta)=\frac{1}{2 \pi} \int_{0}^{\infty} \mathrm{Ai}^{2}\left(\zeta+\zeta^{\prime}\right) \zeta^{\prime} d \zeta^{\prime},
$$

and

$$
\begin{aligned}
\varepsilon_{x}(\zeta)= & \frac{1}{\pi} \int_{-\infty}^{\infty} \int_{0}^{\infty} \int_{0}^{\infty} \operatorname{Ai}(\zeta+\epsilon) \operatorname{Ai}\left(\zeta^{\prime}+\epsilon\right) \operatorname{Ai}\left(\zeta+\epsilon^{\prime}\right) \\
& \times \operatorname{Ai}\left(\zeta^{\prime}+\epsilon^{\prime}\right)\left|\zeta^{\prime}-\zeta\right|^{-3} g \\
& \times\left(\sqrt{\epsilon}\left|\zeta^{\prime}-\zeta\right|, \sqrt{\epsilon^{\prime}}\left|\zeta^{\prime}-\zeta\right|\right) d \zeta^{\prime} d \epsilon d \epsilon^{\prime} .
\end{aligned}
$$

A contour plot of the universal function $g\left(s, s^{\prime}\right)$ may be found in Ref. 24. The exchange energy (4) may be written in the form

$$
\varepsilon_{x}(z)=\varepsilon_{x}^{L D A}(z) F_{x}(s(z)),
$$

where $\varepsilon_{x}^{L D A}(z)$ is the exchange energy density of the uniform electron gas. The enhancement function

$$
F_{x}(\zeta) \equiv \frac{2}{3}\left(\frac{\pi}{3}\right)^{1 / 3} \frac{\varepsilon_{x}(\zeta)}{n^{4 / 3}(\zeta)}
$$

is the unique function $\left[F_{x}(\zeta), s(\zeta)\right]$ of the scaled gradient

$$
s(\zeta) \equiv \frac{n^{\prime}(\zeta)}{2\left(3 \pi^{2}\right)^{1 / 3} n^{4 / 3}(\zeta)}
$$

plotted in Fig. 1. For comparison we also present results obtained by the GGA of Perdew, Burke, and Ernzerhof (PBE) as defined in Ref. 5, and the second order gradient expansion $^{32}$ (GEA). It follows from the figure that the exchange density (7) in the low-gradient limit of the Airy gas model reduces to $\varepsilon_{x}^{L D A}(z)$ as it should. In the large gradient

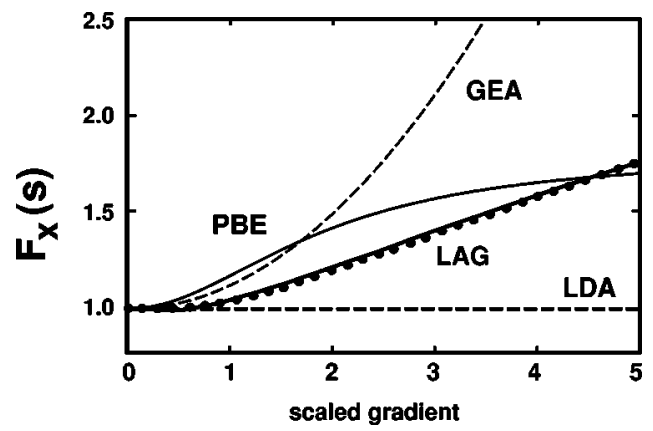

FIG. 1. The exchange energy enhancement function (8) and the parametrized form (11), the latter indicated by dots, of the local Airy gas (LAG) compared to those of the local density approximation (LDA), the generalized gradient approximation (PBE) as defined in Ref. 5, and the second-order gradient expansion (GEA) (Ref. 32).

limit $\varepsilon_{x}(\zeta) \approx-n(\zeta) / 2 \zeta$ (Ref. 24) and similar to the case of the kinetic energy density, ${ }^{29}$ we use the properties of the Airy gas to find the following explicit asymptotic expression:

$$
\begin{aligned}
\varepsilon_{x}(n(z)) \approx & -\frac{e^{2}}{2} \frac{n(z)}{2}\left[n(z) \frac{\partial^{3} n(z)}{\partial z^{3}}-\frac{\partial n(z)}{\partial z} \frac{\partial^{2} n(z)}{\partial z^{2}}\right] \\
& \times\left[\frac{\partial n(z)}{\partial z}\right]^{-2}
\end{aligned}
$$

in terms of the density and its derivatives.

The density of the exchange energy per electron of the Airy gas is plotted as a function of the distance $z$ from the electronic edge in Fig. 2. It is seen that the large gradient expression (10) is accurate for $z / l>-1.4$ corresponding to $s>0.5$. It is also seen that neither the LDA nor the PBE GGA (Ref. 5) leads to the correct behavior near and beyond the electronic edge at $z=0$.

The scaled gradient is conserved when going from the real electron gas to the Airy gas model, ${ }^{29}$ and therefore the enhancement function $F_{x}(s)$ parametrized, for instance, in a modified Becke form ${ }^{33}$

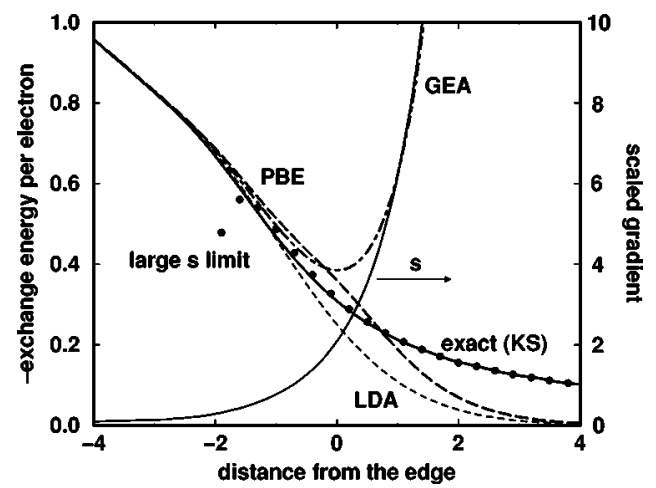

FIG. 2. The exchange energy per electron, $-\varepsilon_{x}(z) / n(z)$, of the Airy gas, obtained from the enhancement factors shown in Fig. 1, plotted as a function of the distance from the electronic edge and compared to the exact result obtained from Eqs. (4) and (6) and the explicit large gradient limit (10). Energy in units of $-\left(e^{2} / 2\right) l^{-1}$ and distance in units of $l$ defined in Eq. (2). The scaled gradient $s$ is also shown. 
TABLE I. The effect of GEA (Ref. 32), PBE (Ref. 5), and LAG gradient corrections (in percentage) on the LDA atomic exchange energies. All functionals are evaluated from the self-consistent LDA (Ref. 31) Kohn-Sham densities. KS denotes the relative difference of the exact and LDA exchange energies from Ref. 13.

\begin{tabular}{lcccc}
\hline \hline Atom & GEA & PBE & LAG & KS \\
\hline $\mathrm{He}$ & 13.9 & 15.0 & 4.2 & 16.0 \\
$\mathrm{Ne}$ & 6.7 & 9.4 & 1.9 & 9.7 \\
$\mathrm{Ar}$ & 5.1 & 7.7 & 1.4 & 8.3 \\
$\mathrm{Kr}$ & 3.5 & 5.5 & 0.9 & 5.9 \\
$\mathrm{Xe}$ & 2.9 & 4.7 & 0.7 & 5.0 \\
\hline \hline
\end{tabular}

$$
F_{x}^{L A G}(s)=1+\beta \frac{s^{\alpha}}{\left(1+\gamma s^{\alpha}\right)^{\delta}},
$$

which includes the proper LDA limit, may be used to obtain the exchange energy density of the real electron gas from the local, scaled gradient $s(n(z))$. For $\alpha=2.626712, \beta$ $=0.041106, \gamma=0.092070$, and $\delta=0.657946$ we find that the local deviation between the exact result (8) and the parametrized form (11) integrated over the range $0<s<20$ is less than $0.3 \%$. We note that the present parametrization, being an overall fit, does not reduce to the GEA (Refs. $15,34)$ in the low-gradient limit. In contrast to the case of the kinetic energy ${ }^{29}$ we have not been able to find an explicit, analytical expression for the exchange energy for small $s$ values, and to establish the behavior numerically has not been attempted because the $s \rightarrow 0$ limit is reached only at $z \rightarrow-\infty$ as seen in Fig. 2. The exact behavior of the LAG exchange functional at $s \rightarrow 0$ is therefore not known at present.

In the following we report the results of applying the LAG exchange functional to four test systems: (i) rare-gas atoms, (ii) diatomic molecules, (iii) jellium surfaces, and (iv) solids. In all cases the total energy is calculated using selfconsistent LDA densities. For molecules and solids the LAG exchange energy is combined with the LDA correlation energy, ${ }^{31}$ since correlation effects have not been worked out in the Airy gas model. The motivation of this combination is given in terms of the enhancement function over the local exchange energy, ${ }^{5}$ defined as $F_{x c}(s) \equiv \epsilon_{x c}[n] / \epsilon^{L D A}(n)$, where $\epsilon_{x c}[n]$ denotes the exchange-correlation energy density.

Most of the currently applied approximate density functionals are based on error cancellations between the exchange and correlation energies. ${ }^{13,26}$ For physically interesting densities this cancellation leads to $F_{x c}(s)$ with negligible slope up to $s \approx 1$. Plots of the enhancement function over the local exchange energy for gradient level and meta-GGA approximations can be found in Refs. 5 and 13. In the present LAG exchange plus LDA correlation scheme this function becomes $F_{x c}^{L A G}(s)=F_{x}^{L A G}(s)+\epsilon_{c}^{L D A}(n) / \epsilon_{x}^{L D A}(n)$, where $\epsilon_{c}^{L D A}(n)$ is the correlation energy density of the uniform electron gas. Thus, the $F_{x c}^{L A G}(s)$ is determined only by the LAG enhancement function (11), which, for $s<1$, is a slowly increasing function of $s$. Therefore, we expect the present exchange-correlation scheme to preserve the excellent cancellation properties of the LDA and PBE GGA, and,
TABLE II. The effect of PBE (Ref. 5) and LAG gradient corrections (in percentage) on the LDA atomization energies for diatomic molecules. Both functionals are evaluated from the selfconsistent LDA (Ref. 31) Kohn-Sham densities generated by the FCD-EMTO (Refs. 35-37). Expt. denotes the relative difference of the experimental and LDA atomization energies from Ref. 13.

\begin{tabular}{cccc}
\hline Molecule & PBE & LAG & Expt. \\
\hline $\mathrm{Li}_{2}$ & -22.6 & -17.8 & 2.1 \\
$\mathrm{Be}_{2}$ & -25.9 & -32.8 & -76.6 \\
$\mathrm{CO}$ & -9.8 & -8.6 & -13.3 \\
$\mathrm{~N}_{2}$ & -12.2 & -10.7 & -14.5 \\
$\mathrm{NO}$ & -14.2 & -11.2 & -23.0 \\
$\mathrm{O}_{2}$ & -16.1 & -16.0 & -31.1 \\
\hline \hline
\end{tabular}

at the same time, to bring the calculated properties in closer agreement with experiment than conventional LDA.

For the rare-gas atoms included in Table I the GEA, PBE, and LAG functionals yield exchange energies, which are, on the average, $6.4 \%, 8.5 \%$, and $1.8 \%$, respectively, larger than those obtained in the LDA. The PBE values are in very good agreement with the exact Kohn-Sham results, ${ }^{13,34}$ which are given relative to the LDA energies in the last column of the table. The LAG approximation represents only a minor improvement relative to the LDA total atomic exchange energies.

The effect of the gradient correction to the LDA atomization energies for a few selected diatomic molecules is shown in Table II which also includes the relative difference between the LDA results and experimental data. ${ }^{13}$ Here, the LDA charge densities for the molecules have been generated using the full charge density (FCD) technique in conjunction with the exact muffin-tin orbital method (EMTO). ${ }^{35-37}$ It is seen that the LAG approximation (i.e., LAG exchange and LDA correlation energy) and PBE GGA have comparable accuracy: Both functionals reduce the LDA overbinding and yield atomization energies, which are, on the average, $16.8 \%$ (PBE) and $16.2 \%$ (LAG) smaller than the LDA values.

In Fig. 3 we compare four exchange functionals applied to the jellium model of metallic surfaces. ${ }^{30}$ The fact that for a given $r_{s}$ value the exchange energies become increasingly negative in the order LDA, LAG, GEA, and PBE is a simple consequence of the enhancement functions shown in Fig. 1

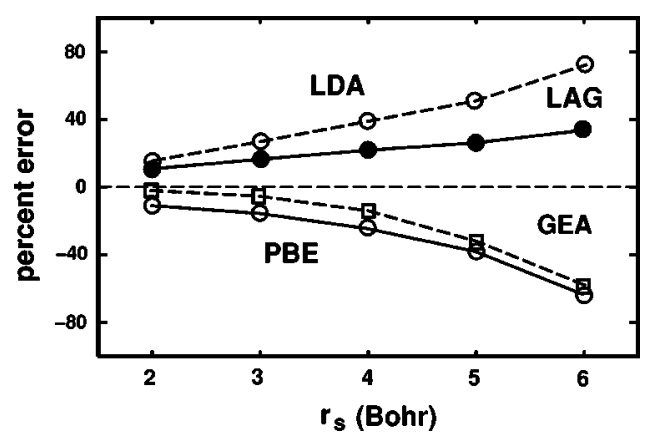

FIG. 3. The exchange energy of the LDA (Ref. 31), GEA (Ref. 32), PBE (Ref. 5), and LAG approximations obtained from the self-consistent Kohn-Sham densities relative to the exact exchange energy (Ref. 40) in the jellium surface model for a range of density parameters $r_{s}=(3 / 4 \pi n)^{1 / 3}$. 


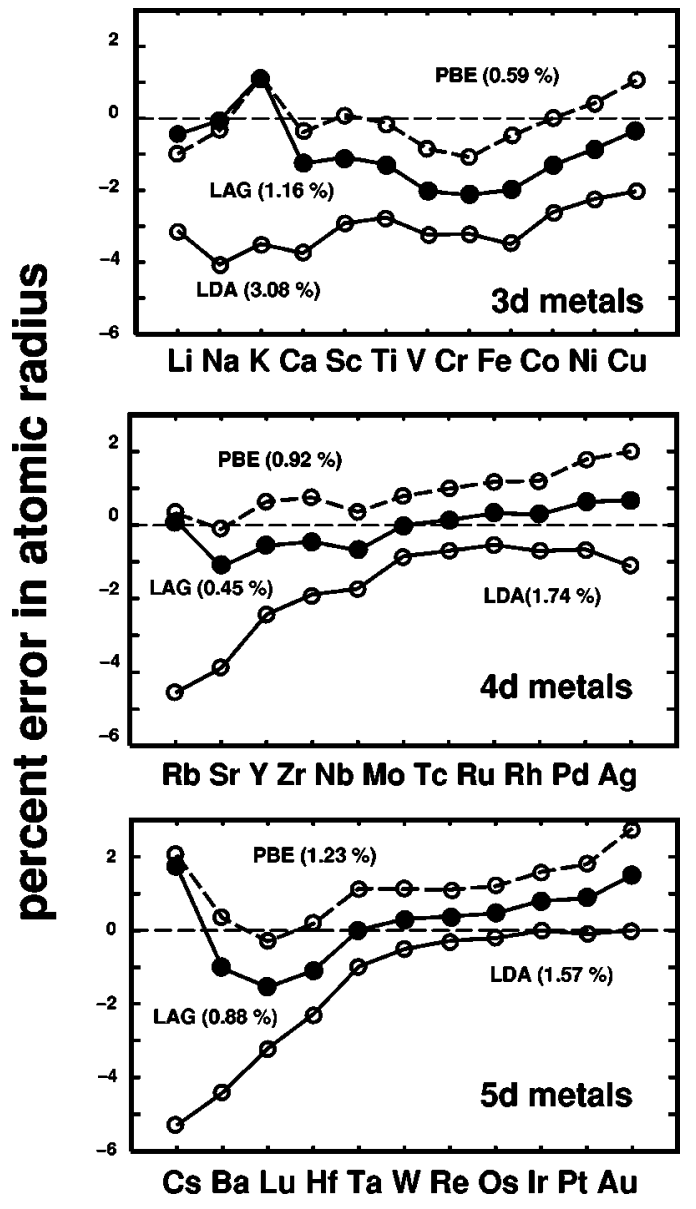

FIG. 4. Relative deviations of the calculated and experimental (Ref. 38) equilibrium atomic radii for the alkali, alkaline earth, $3 d$, $4 d$, and $5 d$ transition metals using LDA (Ref. 31), PBE (Ref. 5), and LAG energy functionals. The numbers in parentheses are the average deviations.

and in agreement with the observation that the GGA significantly underestimate surface exchange energies. ${ }^{15}$ We note that the LAG approximation represents an improvement over both the LDA and PBE and varies less with $r_{s}$ than either of the other two approximations.

As a final test of the LAG approximation we have calculated the atomic volumes and bulk moduli of several metals and semiconductors in their observed low temperature crystal structures by means of the FCD-EMTO method. ${ }^{35-37}$ The results for the equilibrium atomic radii are plotted in Fig. 4. For some selected metals and semiconductors, for which accurate LDA, PBE, and meta-GGA results have been published ${ }^{13}$ the atomic radii and bulk moduli are presented in Table III. The comparison of our LDA ${ }^{31}$ atomic radii for the transition-metal series with those obtained by the fullpotential linear muffin-tin orbital ${ }^{17}$ and linear augmented plane-wave ${ }^{21}$ methods using the same LDA gives mean deviations of $0.33 \%, 0.43 \%$, and $0.49 \%$ for the $3 d, 4 d$, and $5 d$ series, respectively. For $\mathrm{Li}$ and $\mathrm{Na}$ the present LDA results agree within $0.07 \%$ with the full-potential values from Ref. 15. We therefore expect that the results of the present LAG and PBE calculations shown in Fig. 4 will deviate less than $0.5 \%$ from full-potential calculations. The mean deviations between the present atomic radii and bulk moduli listed in Table III and those of Ref. 13 obtained using the linear augmented plane-wave method are $0.20 \%$ and $3.28 \%$ for the LDA and $0.27 \%$ and $3.26 \%$ for the PBE functionals.

The LDA atomic radii shown in Fig. 4 deviate, on average, by $2.26 \%$ from the experimental values, ${ }^{38,39}$ while those calculated in the LAG model and the PBE deviate by $0.83 \%$ and $0.91 \%$, respectively. Among the energy functionals considered in Table III the LAG is found to give the lowest mean deviations for both atomic radii and bulk moduli. We note that for these solids the LAG approximation achieves the accuracy of the recently developed meta-GGA. ${ }^{12,13}$

We have used the Airy gas model of the edge electron gas that is equivalent to the linear potential approximation to develop an exchange energy functional that may serve as an alternative to the functionals based on the generalizedgradient approximation, e.g., PBE GGA. Test calculations for finite and extended systems show that the LAG approximation is more accurate than the local-density approximation in all cases. While the LAG results for atoms are very close to the LDA results, and hence inferior to the PBE GGA

TABLE III. Theoretical equilibrium atomic radii (in bohr) and bulk moduli (in GPa) for some selected solids. The present calculations have been performed for crystallographic $\alpha$ phases using the FCD-EMTO method (Refs. 35-37). The results obtained by the meta-GGA of Perdew, Kurth, Zupan, and Blaha (PKZB) and the experimental values are from Ref. 13. The mean absolute values of the relative errors are shown in parentheses.

\begin{tabular}{lcccccccccc}
\hline \hline & $S_{L D A}$ & $S_{P B E}$ & $S_{L A G}$ & $S_{P K Z B}$ & $S_{\text {Expt. }}$ & $B_{L D A}$ & $B_{P B E}$ & $B_{L A G}$ & $B_{P K Z B}$ & $B_{\text {Expt. }}$ \\
\hline $\mathrm{Na}$ & 3.769 & 3.916 & 3.927 & 4.019 & 3.936 & 8.2 & 7.6 & 7.3 & 7.0 & 6.9 \\
$\mathrm{Al}$ & 2.947 & 2.989 & 2.977 & 2.966 & 2.991 & 81.2 & 75.2 & 76.2 & 90.5 & 77.3 \\
$\mathrm{Fe}$ & 2.565 & 2.645 & 2.604 & 2.627 & 2.667 & 253 & 178 & 209 & 198 & 172 \\
$\mathrm{Cu}$ & 2.602 & 2.684 & 2.656 & 2.656 & 2.658 & 193 & 137 & 157 & 154 & 138 \\
$\mathrm{Pd}$ & 2.846 & 2.916 & 2.883 & 2.888 & 2.873 & 235 & 184 & 203 & 181 & 181 \\
$\mathrm{~W}$ & 2.929 & 2.977 & 2.953 & 2.946 & 2.940 & 312 & 292 & 299 & 311 & 310 \\
$\mathrm{Pt}$ & 2.888 & 2.943 & 2.916 & 2.908 & 2.892 & 304 & 244 & 268 & 267 & 283 \\
$\mathrm{Au}$ & 2.998 & 3.081 & 3.043 & 3.041 & 2.997 & 194 & 134 & 156 & 153 & 172 \\
$\mathrm{Si}$ & 3.163 & 3.198 & 3.189 & 3.200 & 3.182 & 100 & 92.8 & 94.0 & 93.6 & 98.8 \\
$\mathrm{Ge}$ & 3.303 & 3.384 & 3.354 & 3.349 & 3.318 & 71.6 & 61.2 & 64.0 & 64.6 & 76.8 \\
$\mathrm{GaAs}$ & 3.296 & 3.375 & 3.346 & 3.347 & 3.312 & 73.0 & 62.0 & 72.1 & 65.1 & 74.8 \\
$\mathrm{NaCl}$ & 3.202 & 3.346 & 3.337 & 3.284 & 3.306 & 32.9 & 23.0 & 21.7 & 28.1 & 24.5 \\
& $(1.48 \%)$ & $(1.28 \%)$ & $(0.80 \%)$ & $(0.88 \%)$ & & $(17.2 \%)$ & $(9.2 \%)$ & $(9.1 \%)$ & $(9.3 \%)$ & \\
\hline \hline
\end{tabular}


results, its accuracy for the atomization energies of diatomic molecules is similar to that of the PBE GGA. In bulk systems the LAG results are, on average, closer to the experimental values than those obtained in the PBE GGA. These results are very satisfactory in view of the fact that the LAG exchange functional is derived solely from the properties of the Airy gas, and, hence, with no a priori assumptions concerning the exchange enhancement factor. In this sense it is truly $a b$ initio but for the correlation effects, which needs to be worked out in the Airy gas model.
We gratefully acknowledge interesting and fruitful discussions with Professor Walter Kohn. L.V. acknowledges the valuable observations by Professor J. P. Perdew and Dr. A. Nagy. L.V. and B.J. are grateful to the Swedish Natural Science Research Council and the Swedish Foundation for Strategic Research for financial support. Part of this work was supported by the research project OTKA 23390 of the Hungarian Scientific Research Fund. The Center for AtomicScale Materials Physics is sponsored by the Danish National Research Foundation.
${ }^{1}$ P. Hohenberg and W. Kohn, Phys. Rev. 136, B864 (1964).

${ }^{2}$ W. Kohn and L. J. Sham, Phys. Rev. 140, A1133 (1965).

${ }^{3}$ J. P. Perdew and Y. Wang, Phys. Rev. B 33, 8800 (1986); J. P. Perdew, ibid. 33, 8822 (1986).

${ }^{4}$ J. P. Perdew, in Electronic Structure of Solids '91, edited by P. Ziesche and H. Eschrig (Akademie Verlag, Berlin, 1991).

${ }^{5}$ J. P. Perdew, K. Burke, and M. Ernzerhof, Phys. Rev. Lett. 77, 3865 (1996).

${ }^{6}$ Y. Zhang and W. Yang, Phys. Rev. Lett. 80, 890 (1998).

${ }^{7}$ J. P. Perdew, K. Burke, and M. Ernzerhof, Phys. Rev. Lett. 80, 891 (1998)

${ }^{8}$ C. Lee, W. Yang, and R. G. Parr, Phys. Rev. B 37, 785 (1988).

${ }^{9}$ A. D. Becke, Phys. Rev. A 38, 3098 (1988).

${ }^{10}$ F. A. Hamprecht, A. J. Cohen, D. J. Tozer, and N. C. Handy, J. Chem. Phys. 109, 6264 (1998).

${ }^{11}$ B. Hammer, L. B. Hansen, and J. K. Norskov, Phys. Rev. B 59, 7413 (1999).

${ }^{12}$ J. P. Perdew, S. Kurth, A. Zupan, and P. Blaha, Phys. Rev. Lett. 82, 2544 (1999)

${ }^{13}$ S. Kurth, J. P. Perdew, and P. Blaha, Int. J. Quantum Chem. 75, 889 (1999)

${ }^{14}$ A. D. Becke, J. Chem. Phys. 84, 4524 (1986).

${ }^{15}$ J. P. Perdew, J. A. Chevary, S. H. Vosko, K. A. Jackson, M. R. Pederson, D. J. Singh, and C. Fiolhais, Phys. Rev. B 46, 6671 (1992).

${ }^{16}$ L. Vitos, J. Kollár, and H. L. Skriver, Phys. Rev. B 55, 13521 (1997).

${ }^{17}$ V. Ozilins and M. Körling, Phys. Rev. B 48, 18304 (1993).

${ }^{18}$ J. Kollár, L. Vitos, and H. L. Skriver, Phys. Rev. B 55, 15353 (1997).

${ }^{19}$ P. Söderlind, J. A. Moriarty, and J. M. Wills, Phys. Rev. B 53, 14063 (1996).

${ }^{20}$ J. H. Cho and M. Scheffler, Phys. Rev. B 53, 10685 (1996).

${ }^{21}$ A. Khein, D. J. Singh, and C. J. Umrigar, Phys. Rev. B 51, 4105 (1995).

${ }^{22}$ A. D. Corso, A. Pasquarello, A. Baldereschi, and R. Car, Phys. Rev. B 53, 1180 (1996).

${ }^{23}$ C. Filippi, D. J. Singh, and C. J. Umrigar, Phys. Rev. B 50, 14 947 (1994).
${ }^{24}$ W. Kohn and A. E. Mattsson, Phys. Rev. Lett. 81, 3487 (1998).

${ }^{25}$ M. Städele, J. A. Majewski, P. Vogl, and A. Görling, Phys. Rev. Lett. 79, 2089 (1997); M. Städele, M. Moukara, J. A. Majewski, P. Vogl, and A. Görling, Phys. Rev. B 59, 10031 (1999).

${ }^{26}$ A. Görling, Phys. Rev. Lett. 83, 5459 (1999).

${ }^{27}$ S. Ivanov, S. Hirata, and R. J. Barlett, Phys. Rev. Lett. 83, 5455 (1999).

${ }^{28}$ A. D. Becke, J. Chem. Phys. 98, 1372 (1993); 98, 5648 (1993); 109, 8188 (1998).

${ }^{29}$ L. Vitos, B. Johansson, J. Kollár, and H. L. Skriver, Phys. Rev. A 61, 52511 (2000)

${ }^{30}$ N. D. Lang and W. Kohn, Phys. Rev. B 1, 4555 (1970).

${ }^{31}$ J. Perdew and Y. Wang, Phys. Rev. B 45, 13244 (1992).

${ }^{32}$ Here we use the second-order gradient expansion enhancement factor, $1+C_{x} s^{2}$, with $C_{x}=0.1234$, which according to Ref. 34, p. 87, should be preferred over the original $C_{x}=0.0864$. In fact, we find that the original value performs better for both the Airy gas and jellium models.

${ }^{33}$ A. D. Becke, J. Chem. Phys. 85, 7184 (1986).

${ }^{34}$ R. M. Dreizler and E. K. U. Gross, Density Functional Theory (Springer-Verlag, Berlin, 1990), and references therein.

${ }^{35}$ J. Kollár, L. Vitos, and H. L. Skriver, in Electronic Structure and Physical Properties of Solids: The uses of the LMTO Method, edited by H. Dreyssé (Springer-Verlag, Berlin, 2000), p. 85113.

${ }^{36}$ O. K. Andersen, O. Jepsen, and G. Krier, in Lectures on Methods of Electronic Structure Calculations, edited by V. Kumar, O. K. Andersen, and A. Mookerjee (World Scientific, Singapore, 1994), pp. 63-124.

${ }^{37}$ L. Vitos, H. L. Skriver, B. Johansson, and J. Kollár, Comput. Mater. Sci. 18, 24 (2000).

${ }^{38}$ D. A. Young, Phase Diagrams of the Elements (University of California Press, Berkeley, 1991).

${ }^{39}$ The radii taken from Ref. 38 are reduced to $T=0 \mathrm{~K}$ using the linear thermal expansion coefficients from W. B. Pearson, A Handbook of Lattice Spacings and Structures of Metals and Alloys (Pergamon Press, London, 1958).

${ }^{40}$ The exact Kohn-Sham jellium surface exchange energies are estimated using the LDA and the exact results from Table II of A. R. E. Mohammed and V. Sahni, Phys. Rev. B 31, 4879 (1985). 